\title{
Face Recognition System on Raspberry Pi
}

\section{Olegs Nikisins, Rihards Fuksis, Arturs Kadikis, Modris Greitans}

\author{
Institute of Electronics and Computer Science. 14 Dzerbenes Street, Riga, LV 1006, Latvia
}

\author{
Keywords: Face recognition, Raspberry Pi, Local Binary Pattern.
}

\begin{abstract}
An embedded face recognition system based on the Raspberry Pi single-board computer is proposed in this paper. Face recognition system consists of face detection and face localization using Haar feature-based cascade classifier. Face features are extracted using weighted Local Binary Pattern algorithm. Developed system performs one full face analysis in $110 \mathrm{~ms}$. Comparison of two biometric samples is performed in $2 \mathrm{~ms}$. The proposed embedded face recognition system was tested on FERET database and achieves accuracy of CMC: $99.33 \%$ and EER: $1 \%$.
\end{abstract}

\section{Introduction}

Real time human identification systems are important for security, surveillance and biometric applications. Usually it is desirable to detect, track and recognize persons in public areas such as airports, shopping centres, in areas with restricted access such as private offices, houses etc. Human identification can be performed by analysing its biometric information, such as fingerprints, face, iris, palm prints, palm veins etc. However, for fast and convenient person recognition, still the most suitable biometric parameter is facial information.

Identification of humans by using facial biometrics is still challenging task, due to the variable illumination, changing facial expressions according to mood changes, head orientation and pose. Over the years, various face detection algorithms have been developed. Some face recognition methods analyse the geometric features of facial images, such as location and distance between nose, eyes, and mouth [5] [3]. However, these methods are sensitive to the changes in illumination and facial expression. Because of this drawback, most of the face recognition systems try to extract some holistic features from the original face images for matching. By using holistic methods face is recognized using descriptions based on the entire image rather than on local features of the face [6]. Many subspace learning based holistic feature extraction methods have been developed, including Eigenfaces [12], Fisherfaces [2], 2D PCA [14] and others.

In this paper we describe the holistic method called local binary pattern (LBP) [9]. In this paper we propose an embedded face recognition system that can be used as a system to control electromagnetic door lock of the doors, to recognize persons at boarder or elsewhere.

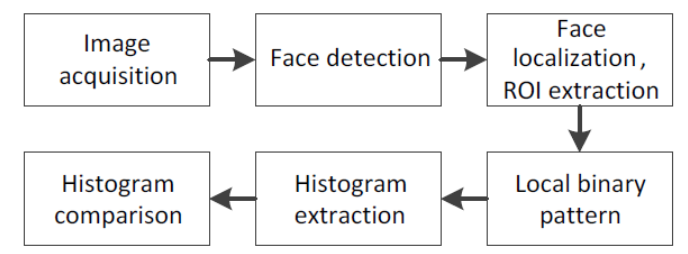

Fig. 1. Face recognition steps

The paper is divided in two main parts: section 2 explains the face detection and recognition algorithm and section 3 explains the implementation and the information about how the system was built. At the end we introduce the experimental setup and the results and give conclusions about this work.

\section{Face recognition algorithm}




\begin{tabular}{|l|l|}
\hline$A$ & $B$ \\
\hline$C$ & $D$ \\
\hline \multicolumn{2}{|l}{} \\
\hline
\end{tabular}

Fig. 2. The sum of the pixels within rectangle $\mathrm{D}$ can be computed with four array references. $(A+B+C+D)+(A)-((A$ $+B)+(A+C))$

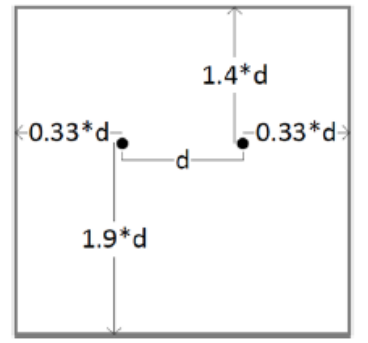

Fig. 3. Coefficients for face region extraction

The proposed algorithm for face recognition can be divided into several steps. The sequence of steps of the proposed algorithm used for face recognition is shown in Fig. 1. The first step is to acquire the image. Next, face detection has to be performed, to find whether the face appears in the captured image or not. The next step is to locate the position of the face in the image. Face detection and face localization is per- formed by using Haar feature-based cascade classifier [8] [9]. The rectangular features needed for Haar classifier are computed using an intermediate representation for the image that is called an integral image [8].

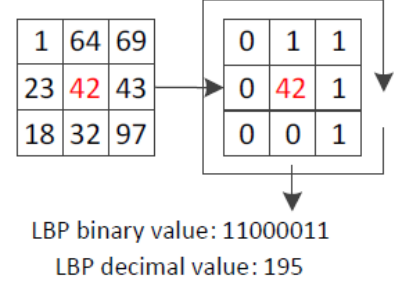

Fig. 4. LBP example of $3 \times 3$ neighborhood, $(P=8, R=1)$

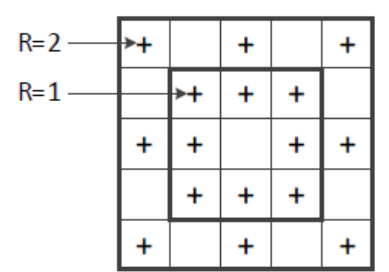

Fig. 5. Labelling principle

The integral image at location $x ; y$ contains the sum of the pixels above and to the left of $x ; y$, inclusive:

$$
i(x, y)=\sum_{x^{\prime} \leq x, y^{\prime} \leq y} f\left(x^{\prime}, y^{\prime}\right)
$$

, where $i(x, y)$ is the integral image and $f(x, y)$ is the original image. Using the following pair of recurrences:

$$
\begin{gathered}
s(x, y)=s(x, y-1)+f(x, y) \\
i(x, y)=i(x-1, y)+s(x, y)
\end{gathered}
$$

(where $s(x, y)$ is the cumulative row sum, $s(x,-1)=0$, and $i(-1 ; y)=0$ ) the integral image can be computed in one pass over the original image [8].

Using the integral image any rectangular sum can be computed by referencing four array locations (shown in Fig. 2). Difference between two rectangular sums can be computed in eight references. Since the two-rectangle features defined above involve adjacent rectangular sums they can be computed in six array references, eight in the case of the three- rectangle features, and nine for fourrectangle features [8].

If the image contains a face, the algorithm returns a rectangle with coordinates where face was found. However, it is not the final region of interest (ROI) that we use. To calculate the necessary ROI, we use the coordinates of a rectangle and recalculate the ROI position. We use FERET face image database for algorithm evaluation that contains many frontal face images with their respective eye coordinates. Using the obtained rectangle from the Haar classifier and the known eye positions, we developed a method to statistically determine where in the image the eyes are most likely to be located. 


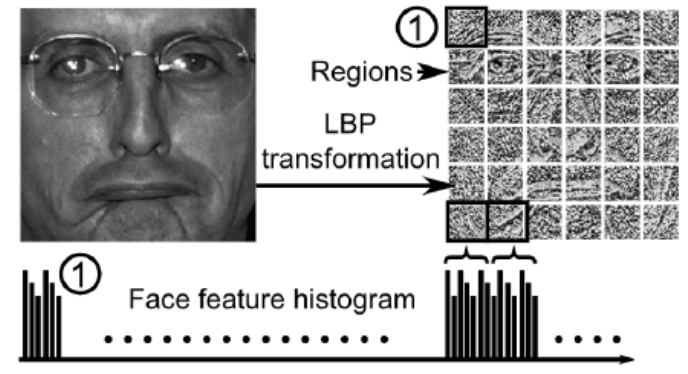

Fig. 6. Example of LBP transform and formation of face feature histogram from $6 x 6$ regions

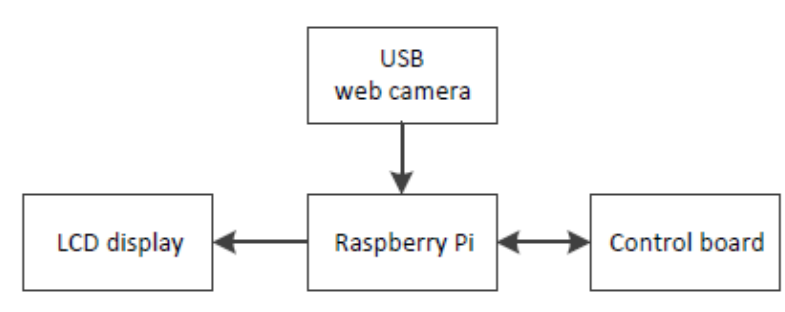

Fig. 7. Hardware block scheme

We use this method to determine the approximate location of the person's eyes to calculate the ROI based of the distance between the eyes d. Distance to each of the sides is calculated by coefficients shown in Fig. 3. However, our approach does not localize the position of the eyes and therefore cannot correct the rotations of the face.

After the ROI is found, we perform LBP transformation. LBP labels each pixel by thresholding the neighbourhood pixels with central pixel value and represents the result as a bi- nary number. An example of the labelling procedure for $3 \times 3$ region is shown in Fig. 4. Binary value usually is written as a decimal number and stored in the centre pixel position of the output image. After LBP transform a histogram of labels is used as a descriptor of the image. LBP operator can be performed by using different amount of pixels $\mathrm{P}$ on the sampling circle and different radius $\mathrm{R}$ from the central pixel. Example of radius $R=1$ and $R=2$ with $P=8$ is shown in Fig. 5. A histogram of the labelled image $f_{l}(x ; y)$ can be calculated as follows:

$$
H_{i}=\sum_{x, y} I\left\{f_{l}(x, y)=i\right\}, i=0, \ldots, n-1,
$$

where $n=2^{P}$ is the number of different labels and

$$
I\{A\}= \begin{cases}1, & A=\text { true } \\ 0, & A=\text { false }\end{cases}
$$

In order to save the spatial information about the object, LBP transformed image is divided into several sub-regions $R_{0}, R_{1}, \ldots, R_{m-1}$. A spatially enhanced histogram is calculated by concatenating the region histograms into a single feature histogram:

$$
H_{i, j}=\sum_{x, y} I\left\{f_{l}(x, y)=i\right\}, I\left\{(x, y) \in R_{j}\right\}
$$

An example of LBP transform and face feature histogram forming with $6 x 6$ regions of LBP image is shown in Fig. 6.

Feature histogram is used as a descriptor for each of the given images. To compare two feature histograms many methods can be used. For example, correlation of the histograms, Chi-Square [10], histogram intersection [11], Bhattacharyya distance [12] and other methods. In our algorithm we chose histogram intersection for two histogram comparison. Research on using LBP transform for face recognition from which this implementation was derived is published in [13].

\section{Face recognition system}

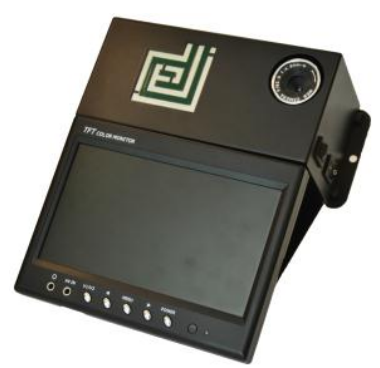

Fig. 8. Developed face recognition system 
In the heart of the embedded face recognition system is a Raspberry Pi single-board computer, which controls all of the peripherals. The board has a 700MHz ARM CPU and $512 \mathrm{MB}$ of RAM. For image acquisition we use a USB web camera with a 320x240 resolution. To visualize the information and provide user friendly identification procedure, we use mini LCD. A control PCB is developed for voltage con- version and also for electrical door magnet control. Block diagram of the system is shown in Fig. 7. As an enclosure we have developed a custom-made metal enclosure for Rasp- berry Pi, web camera, control board and mounting of LCD. Developed system is shown in Fig. 8. Raspberry Pi runs the Raspbian OS which is a Linux operating system derived from Debian. Our program is written in $\mathrm{C}++$ and uses the OpenCV library for image acquisition and face detection. The developed recognition software can be used as a standalone or a part of a multimodal biometric system. We have developed the data exchange interface so the system could connect to other biometric system to ex- change biometric data. When connected with another biometric system, face recognition system can work as a slave module in capture-send mode. In slave mode system waits for the start signal, performs image acquisition and processing and then sends the acquired data to the master system. Biometric research group has developed the palmprint and palm vein biometric system that acts as a master and controls the face recognition system [14].

\section{Experimental setup and results}

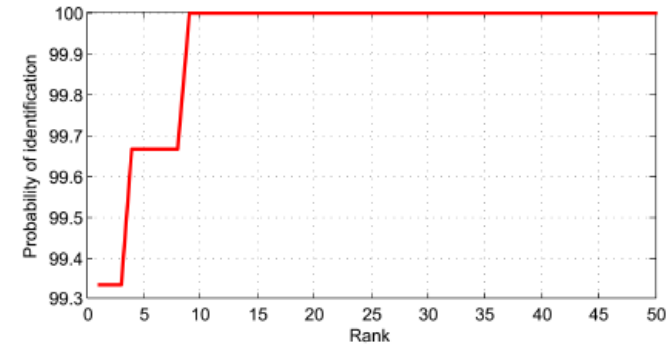

Fig. 9. Cumulative Match Characteristic

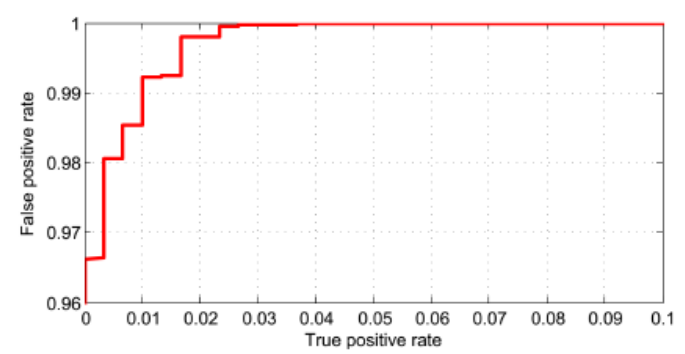

Fig. 10. Receiver Operating Characteristic

For evaluation of performance we use two parameters. One is equal error rate (ERR). The rate at which both accept and reject errors are equal. The value of the EER can be easily obtained from the receiver operating characteristic (ROC) curve. The EER is a quick way to compare the accuracy of devices with different ROC curves. The lower the rate, the lower the error of the system. The other one is cumulative match characteristic (CMC), a CMC curve plots the probability of identification against the rank. The greater the rate at rank 1, the higher the accuracy of the system.

To evaluate the performance of the system we use publicly available FERET database. Probe set and gallery set form the FERET database are combined. The combined data contains data from 992 persons, 2 samples from each person, for a total of 1986 images. $25 \%$ of persons are used for the algorithm training and the other $75 \%$ are used for testing. During the training process, the optimal values for three parameters were found by performing the exhaustive search. The first parameter is the resolution of the ROI, the second one is the radius for the LBP transform and the third one is the amount of regions for the histogram calculation. Optimal parameters are ROI width $=110, R=4, m$ $=5$.

Using the FERET database we achieved the results of CMC: $99.33 \%$ and EER: $1 \%$. CMC curve is shown in Fig. 9 and ROC curve is shown in Fig. 10.

\section{Conclusion}

Embedded face recognition system based on Raspberry Pi single-board computer is introduced in this paper. Paper is divided in two parts - the software part and the hardware part. Software part describes the algorithms for face detection, localization, feature extraction and recognition. Hardware part describes how the system was built and what modules does it use. System was built with an option to connect with other biometric systems such as palmprint and palm vein biometric 
system, which is described in [14]. By combining several biometric parameters we can obtain higher accuracy of identification of authentication and also higher resistance to counterfeiting, because it is always harder to falsify 3 or more parameters than just one. We have also developed a smart card applet that can store and compare biometric data. If biometric data is compared on the card (Match on Card principle) and it is also encrypted we can ensure that the original data is always kept on the card and won't be set outside, therefore, eliminating the chance of data interception.

\section{References}

[1] Ingemar J. Cox, J. Ghosn, and P.N. Yianilos, "Feature- based face recognition using mixturedistance," in Com- puter Vision and Pattern Recognition, 1996. Proceed- ings CVPR '96, 1996 IEEE Computer Society Confer- ence on, 1996, pp. 209-216.

[2] Paola Campadelli and Raffaella Lanzarotti, "A face recognition system based on local feature characteri- zation.," in Advanced Studies in Biometrics, Massimo Tistarelli, Josef Bign, and Enrico Grosso, Eds. 2003, vol. 3161 of Lecture Notes in Computer Science, pp. 147-152, Springer.

[3] Keun-Chang Kwak and W. Pedrycz, "Face recognition using an enhanced independent component analysis ap- proach," Neural Networks, IEEE Transactions on, vol. 18, no. 2, pp. 530-541, 2007.

[4] M. Turk and A. Pentland, "Eigenfaces for recognition," in Journal of Cognitive Neuroscience, 1991, vol. 3, pp. 71-86.

[5] P.N. Belhumeur, J.P. Hespanha, and D. Kriegman, "Eigenfaces vs. fisherfaces: recognition using class spe- cific linear projection," Pattern Analysis and Machine Intelligence, IEEE Transactions on, vol. 19, no. 7, pp. 711-720, 1997.

[6] Jian Yang, D. Zhang, A.F. Frangi, and Jing-Yu Yang, "Two-dimensional pca: a new approach to appearance- based face representation and recognition," Pattern Analysis and Machine Intelligence, IEEE Transactions on, vol. 26, no. 1, pp. 131-137, 2004.

[7] Timo Ojala, Matti Pietikinen, and David Harwood, "A comparative study of texture measures with classifica- tion based on featured distributions," Pattern Recogni- tion, vol. 29, no. 1, pp. $51-59,1996$.

[8] P. Viola and M. Jones, "Rapid object detection using a boosted cascade of simple features," in Computer Vision and Pattern Recognition, 2001. CVPR 2001. Proceed- ings of the 2001 IEEE Computer Society Conference on, 2001, vol. 1, pp. I-511-I-518 vol.1.

[9] R. Lienhart and J. Maydt, "An extended set of haar-like features for rapid object detection," in Image Process- ing. 2002. Proceedings. 2002 International Conference on, 2002, vol. 1, pp. I900-I-903 vol.1.

[10] Ofir Pele and Michael Werman, "The quadratic-chi his- togram distance family," in Computer Vision ECCV 2010, Kostas Daniilidis, Petros Maragos, and Nikos Paragios, Eds. 2010, vol. 6312 of Lecture Notes in Com- puter Science, pp. 749-762, Springer Berlin Heidelberg.

[11] A. Barla, F. Odone, and A. Verri, "Histogram intersec- tion kernel for image classification," in Image Process- ing, 2003. ICIP 2003. Proceedings. 2003 International Conference on, 2003, vol. 3 , pp. III-513-16 vol.2.

[12] Xi Chen and Tat-Jen Cham, "Discriminative distance measures for image matching," in Pattern Recognition, 2004. ICPR 2004. Proceedings of the 17th International Conference on, 2004, vol. 3, pp. 691-695 Vol.3.

[13] Olegs Nikisins, "Weighted multi-scale local binary pat- tern histograms for face recognition," in International Conference on Applied Mathematics and Computational Methods, AMCM 2013, 2013, pp. 76-81.

[14] M. Pudzs, R. Fuksis, R. Ruskuls, T. Eglitis, A. Kadikis, and M. Greitans, "Fpga based palmprint and palm vein biometric system," in Biometrics Special Interest Group (BIOSIG), 2013 International Conference of the, 2013, pp. 1-4. 